\title{
Progress on the study of the anticancer effects of artesunate (Review)
}

\author{
XIULAN YANG ${ }^{1}$, YUDONG ZHENG $^{1}$, LIAN LIU ${ }^{1}$, JIANGRONG HUANG $^{1}$, FEI WANG $^{2}$ and JIE ZHANG ${ }^{1}$ \\ ${ }^{1}$ Department of Pharmacology, The School of Basic Medicine, Health Science Center, Yangtze University, Jingzhou, \\ Hubei 434023; ${ }^{2}$ Center of Experiment and Training, Hubei College of Chinese Medicine, Jingzhou, Hubei 434020, P.R. China
}

Received February 18, 2021; Accepted July 30, 2021

DOI: 10.3892/ol.2021.13011

\begin{abstract}
Artesunate (ART) is a derivative of artemisinin that is extracted from the wormwood plant Artemisia annua. ART is an antimalarial drug that has been shown to be safe and effective for clinical use. In addition to its antimalarial properties, ART has been attracting attention over recent years due to its reported inhibitory effects on cancer cell proliferation, invasion and migration. Therefore, ART has a wider range of potential clinical applications than first hypothesized. The aim of the present review was to summarize the latest research progress on the possible anticancer effects of ART, in order to lay a theoretical foundation for the further development of ART as a therapeutic option for cancer.
\end{abstract}

\section{Contents}

1. Introduction

2. Source and activity of ART

3. Anticarcinogenic mechanism of ART

4. Potential role of ART in human malignancies

5. Summary and perspectives

Correspondence to: Professor Lian Liu, Department of Pharmacology, The School of Basic Medicine, Health Science Center, Yangtze University, 1 Nanhuan Road, Jingzhou, Hubei 434023, P.R. China

E-mail: liulian@yangtzeu.edu.cn

Abbreviations: ART, artesunate; LDH, lactate dehydrogenase; ROS, reactive oxygen species; HCC, hepatocellular carcinoma; TCTP, translation-controlled tumor protein; NSCLC, non-small cell lung cancer; ATC, anaplastic thyroid carcinoma; HNC, head and neck cancer; MPNST, malignant peripheral nerve sheath tumor; EC, esophageal cancer; CRC, colorectal cancer; AR, androgen receptor

Key words: artesunate, artemisinin, antitumor, apoptosis, proliferation

\section{Introduction}

Cancer is a major health concern worldwide $(1,2)$. According to GLOBOCAN 2020, which presented the latest estimates of cancer incidence and mortality (3), there were $\sim 19.3$ million new cases of cancer and 10 million cancer-related deaths worldwide in 2020. As such, the number of cancer cases worldwide is expected to reach 28.4 million by 2040 , a $47 \%$ increase from 2020 (4). Asia, Latin America, the Caribbean and Africa are expected to experience particularly large increases in cancer morbidity and mortality rates (3). Therefore, it is crucial to develop novel anticancer agents.

Artesunate (ART) is a derivative of artemisinin that is characterized by high efficacy, rapid effects, low toxicity and reduced susceptibility to drug resistance $(5,6)$. At present, ART is commonly used for the treatment of mild to severe malaria worldwide (7). However, accumulating evidence has shown that ART also displays anticancer properties, in addition to its antimalarial effect (8). For instance, ART has been reported to induce apoptosis and autophagy in human bladder cancer cells $(9,10)$. Moreover, it can induce cell cycle arrest, reactive oxygen species (ROS) generation and ferroptosis in renal cell carcinoma (11). In the present review, the potential anticancer effects of ART and the underlying mechanism of action involved are summarized. The aim was to provide a theoretical basis for the further development of ART and its derivatives for the treatment of cancer.

\section{Source and activity of ART}

ART is a semi-synthetic, monomeric derivative of artemisinin isolated from Artemisia annua in the 1970s (12-14). The conversion from artemisinin to ART is a two-step process, starting with reduction of dihydroartemisinin with diisobutylaluminium hydride, followed by esterification with succinic anhydride (14). The chemical name of ART is dihydroartemis inin-1,2- $\alpha$-succinate monoester, with the chemical formula of $\mathrm{C}_{24} \mathrm{H}_{39} \mathrm{O}_{8}$ and a molecular weight of $455.56 \mathrm{~g} / \mathrm{mol}$ (15).

ART has a hydrophilic group, and the 1,2,4-endoperoxide bridge is responsible for the antimalarial activity of the drug. ART acts on all stages of malaria parasite circulation. ART also may penetrate the cell membranes and generate ROS, and a small amount of ART reaches the mitochondria of the parasite, where ART and ROS react with each other, leading 
to mitochondrial dysfunction (8). ART is the only artemisinin derivative with high water solubility, due to the addition of the hemisuccinate group. ART is metabolized to docosahexaenoic acid (DHA) as it enters the body (16-18). ART induces the generation of ROS, increasing malondialdehyde levels and decreasing the levels antioxidants such as superoxide dismutase and catalase, thereby causing alkylation of the proteins of the Plasmodium parasite (19). At present, ART is mainly used for the treatment of malaria of all types (20), for immune regulation (in type 1 diabetes in NOD mice) (21), as well as for liver (22), breast (23) and lung (24-26) cancer.

\section{Anticarcinogenic mechanism of ART}

There is considerable evidence that ART can exert anticancer effects on several types of cancer cells $(6,15)$. ART has been reported to induce apoptosis, differentiation and autophagy in colorectal cancer cells by impairing angiogenesis (27), inhibiting cell invasion and migration (28), inducing cell cycle arrest (11), upregulating ROS levels, regulating signal transduction [for example, activating the AMPK-mTOR-Unc-51-like autophagy activating kinase (ULK1) pathway in human bladder cancer cells] (9) and blocking immune escape (29). In addition, ART has been shown to restore the sensitivity of a number of cancer types to chemotherapeutic drugs by modulating various signaling pathways; for example, ART can improve the apoptosis of HCC by inhibiting the PI3K/AKT/mTOR pathway (30), and can increase liver cancer cell sensitivity to sorafenib via suppression of the MEK/ERK pathway (31) (Fig. 1).

Apoptosis. Apoptosis is a type of programmed cell death that does not elicit inflammatory responses (32). A number of studies have shown that ART can induce apoptosis by activating the mitochondria-dependent pathway, specifically by mediating the activation of caspase- 3 and -9 and the release of cytochrome $\mathrm{c}$ into the cytosol after permeabilization of the mitochondrial membrane (33). Additionally, ART can induce HL-60 human acute promyelocytic leukemia cell and KG1a acute myeloid leukemia cell death by regulating antiapoptotic proteins, such as Bcl-2, as well as proapoptotic proteins, such as Bid and Bak, through inhibition of the MEK/ERK and PI3K/Akt pathways (34). ART has also been demonstrated to induce T helper 1 cell differentiation and promote apoptosis in ovarian cancer cells via the microRNA (miR)-142/sirtuin 1 pathway (35).

Autophagy. Autophagy is a conserved, self-degrading system that is essential for maintaining cell homeostasis under stress conditions, and which has been demonstrated to serve an important role in cancer in association with a family of autophagy-related proteins (LC3B) (36). ART can induce autophagy and increase the levels of CD155 in uterine corpus endometrial carcinoma (UCEC) cells. Moreover, it also regulates the interaction between $\mathrm{CD} 155$ and its receptor on the NK92 natural killer cell line by upregulating the co-stimulator CD226 and downregulating the co-inhibitor TIGIT, thereby enhancing the cytotoxicity of these cells. Thus, ART has a dual anticancer effect on UCEC cells (37). ART also induces autophagy by upregulating ROS production and activating the AMP-activated protein kinase/mTOR/ULK1 pathway in human bladder cancer cells (9).
ROS. ROS have a dual role in cellular metabolism (38). Their production is impaired during normal cellular homeostasis, whilst excessive production can lead to oxidative stress (OS), a process that can lead to damage to cellular structure (39). A study has shown that higher levels of ROS are important for the initiation, progression, angiogenesis and metastasis of cancer (40). Dysregulation of ROS has been found to promote tumorigenesis through activation of various oncogenic, signaling pathways such as MAPK, PI3K/AKT/mTOR and $\mathrm{NF}-\kappa \mathrm{B}(18,40)$, DNA damage $(41,42)$, immune escape, metastasis, angiogenesis and telomere elongation (40). ROS production has been demonstrated to play an important role in ART-induced apoptosis in various tumor cell lines, including glioblastoma (43), lymphoma (44), breast cancer cells (45). Yao et al (46) suggested that ART could increase ROS levels in the hepatocellular carcinoma (HCC) cell lines Huh7 and Hep3B. In addition, the combination of sorafenib and ART treatment was found to synergistically produce antiproliferative effects in HCC cells and induce apoptosis.

Inhibition of angiogenesis. Blood vessels provide oxygen and a nutrient supply for the growth of tumors, which also facilitate the proliferation, migration and subsequent invasion of malignant tumor cells in the long term (47). Angiogenesis is a dynamic and complex process that is regulated by a variety of mechanisms. Inhibition of angiogenesis has become a therapeutic strategy for pancreatic cancer (48), breast cancer (49) and ovarian cancer (50). Chen et al (51) demonstrated that ART could downregulate the expression of VEGF and angiopoietin-1 in RPMI8226 myeloma cells, decrease the activation of ERK1and inhibit angiogenesis. Their study indicated that ART possessed a potential anti-myeloma effect, which was mediated by the inhibition of angiogenesis.

Cell cycle arrest. Aberrant cell division is one of the characteristic features of cancer cells (52). ART inhibits the proliferation of bladder cancer cells (RT4, RT112, T24 and TCCSup), which is associated with $\mathrm{G}_{0} / \mathrm{G}_{1}$-phase cell cycle arrest and downregulation of cell cycle regulatory proteins [cyclin D1 and CDK4 (required for entry into the $\mathrm{G}_{1}$ phase); CDK1 and cyclin $\mathrm{A} / \mathrm{B}$ (essential during the late $\mathrm{S}$ phase and early $\mathrm{M}$ phase)] (10). ART can block cell cycle progression and lead to a significant reduction in the levels of the cell cycle activating proteins cyclin $A$, cyclin $B$, and CDK 1 , evoking $G_{0} / G_{1}$ phase arrest and inhibiting growth of the cells in renal cell carcinoma (11). In breast cancer cells (MCF-7 and MDA-MB-231), ART can block $\mathrm{G}_{2} / \mathrm{M}$ progression by upregulating beclin-1 expression, which promotes autophagy (53). In glioblastoma cells (A172, U251 and U87), ART also increases the proportion of cells in the $G_{0} / G_{1}$ phase, reduces the proportion of cells in the $S$ phase and inhibits proliferation by downregulating the expression levels of the cell cycle-related proteins CDK2, CDK4, cyclin D1 and cyclin B1 (54).

Ferroptosis. Ferroptosis is a recently identified form of regulated cell death, which is characterized by iron overload, lipid ROS accumulation and lipid peroxidation (55). Evidence suggests that ferroptosis is closely associated with the occurrence, development and inhibition of cancer (56). Zhang et al (26) demonstrated that ART could upregulate the mRNA levels of transferrin 


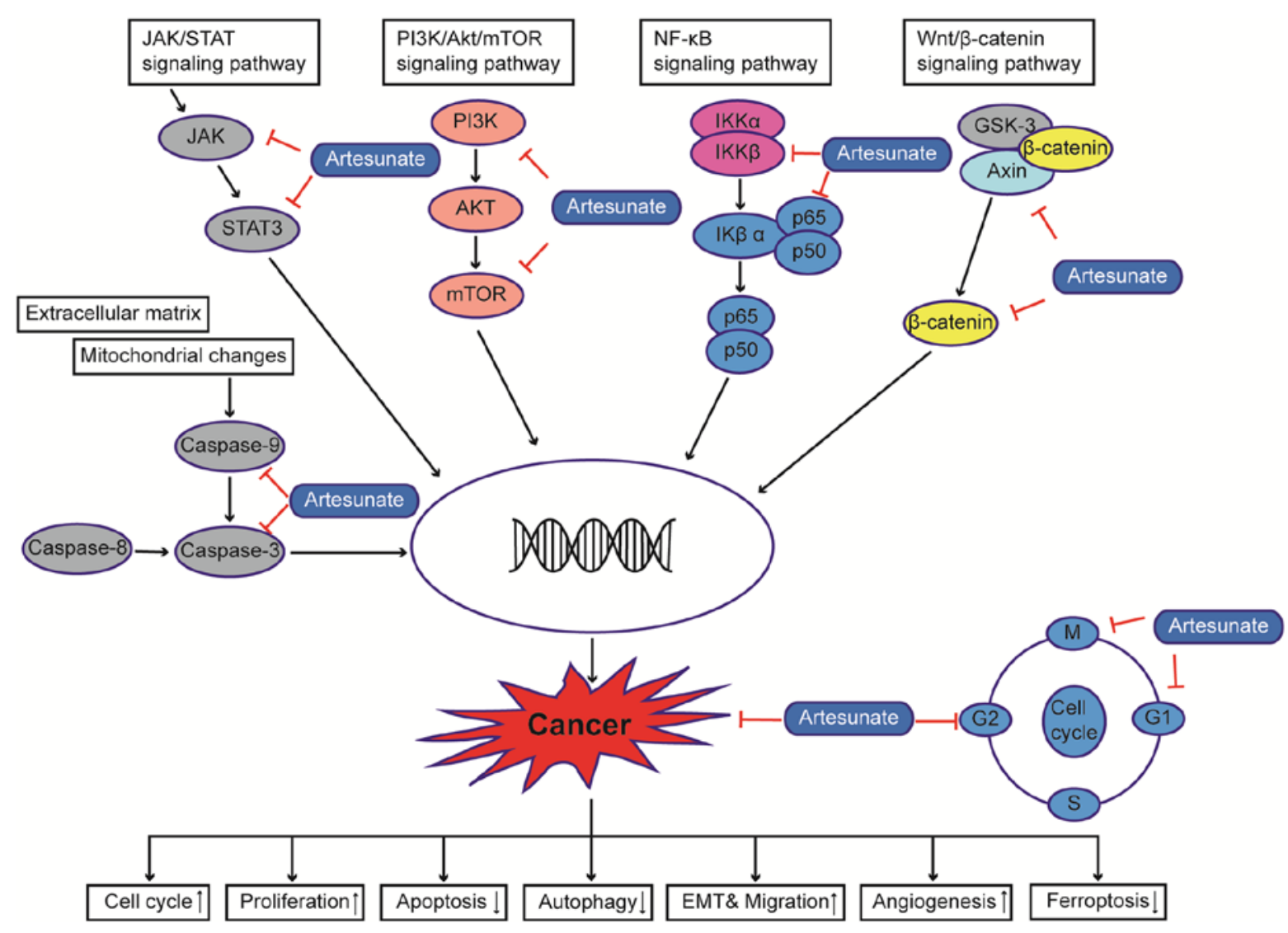

Figure 1. Anticarcinogenic mechanism of artesunate. Multiple molecular and signaling pathways regulate abnormal cell proliferation and migration, such as the NF- $\mathrm{kB}$ signaling pathway, the PI3K/Akt/mTOR signaling pathway and the JAK/STAT signaling pathway, among others, ultimately leading to tumorigenesis. Artesunate may affect the development of cancer by interfering with the cell cycle, proliferation, invasion, angiogenesis and apoptosis of cancer cells by acting on different sites. EMT, epithelial-mesenchymal transition.

receptor (a positive regulator of ferroptosis), thus inducing apoptosis and ferroptosis in A549 non-small cell lung cancer (NSCLC) cells. Li et al (57) showed that ART enhanced the anticancer effects of low-dose sorafenib (a novel multi-targeted oral drug for the treatment of gastroenteric tumors) against Huh7, SNU-449, and SNU-182 HCC cell lines in vitro and against a Huh7 cell xenograft model in BALB/c nude mice. In addition, ART-induced lysosome activation synergizes with the pro-oxidative effects of sorafenib to sequentially promote lysosomal cathepsin $\mathrm{B} / \mathrm{L}$ activation, ferritin degradation, lipid peroxidation and ferroptosis (57).

\section{Potential role of ART in human malignancies}

Previous studies have reported that ART exerted minimal toxicity, was cost-effective and was effective for treating different types of cancer (Table I) (58-78). The potential anticancer properties of ART in different types of cancer are discussed below.

Leukemia. Leukemia is a clonal hematopoietic stem cell malignancy (79). ART can induce leukemic $\mathrm{T}$ cell apoptosis by promoting the generation of mitochondrial ROS (80). Previous studies have suggested that ART induces caspase-3/9-mediated apoptosis by targeting the outer mitochondrial membrane, leading to the activation and nuclear translocation of mitochondrial pro-apoptotic factors in human SKM-1 myelodysplastic syndrome cells (81). Nuclear translocation of apoptosis-inducing factors and endonuclease $G$ were accompanied by low levels of ROS and increased mitochondrial production of superoxide, which occur prior to apoptosis and appear to be associated with the intracellular levels of divalent iron $(59,60,82-84)$. Chen et al (34) found that ART may inhibit the levels of phosphorylated (p)-PI3K, p-AKT, p-MEK1 and p-ERK $1 / 2$ and promote the apoptosis of leukemia cells (HL-60 and KG1a cells) by inactivating PI3K/Akt and MEK/ERK signaling, ART also significantly reduced the expression of Ki67 and survivin, inhibited growth and stemness in KG1 xenograft models (34). In the MV4-11 cell line, ART combined with bortezomib (which is commonly used for the treatment of patients with multiple myeloma) resulted in significantly higher proliferation inhibition and reduced apoptotic rates compared with ART or bortezomib alone in the same concentration gradient. After the combination of the two drugs for $24 \mathrm{~h}$, the expression of the pro-apoptotic proteins BIM and cleaved activated caspase- 3 and the autophagy-related protein LC3B was upregulated in MV4-11 cells, whereas that of the anti-apoptotic protein Bcl-2 was downregulated (58).

Nervous system tumors. Central nervous system tumors comprise a group of malignancies that originate from tissues or structures of the central nervous system and exhibit an incidence of 5.6 per 100,000 person-years in children under the age of 19 (85-87). ART can selectively downregulate the expression 


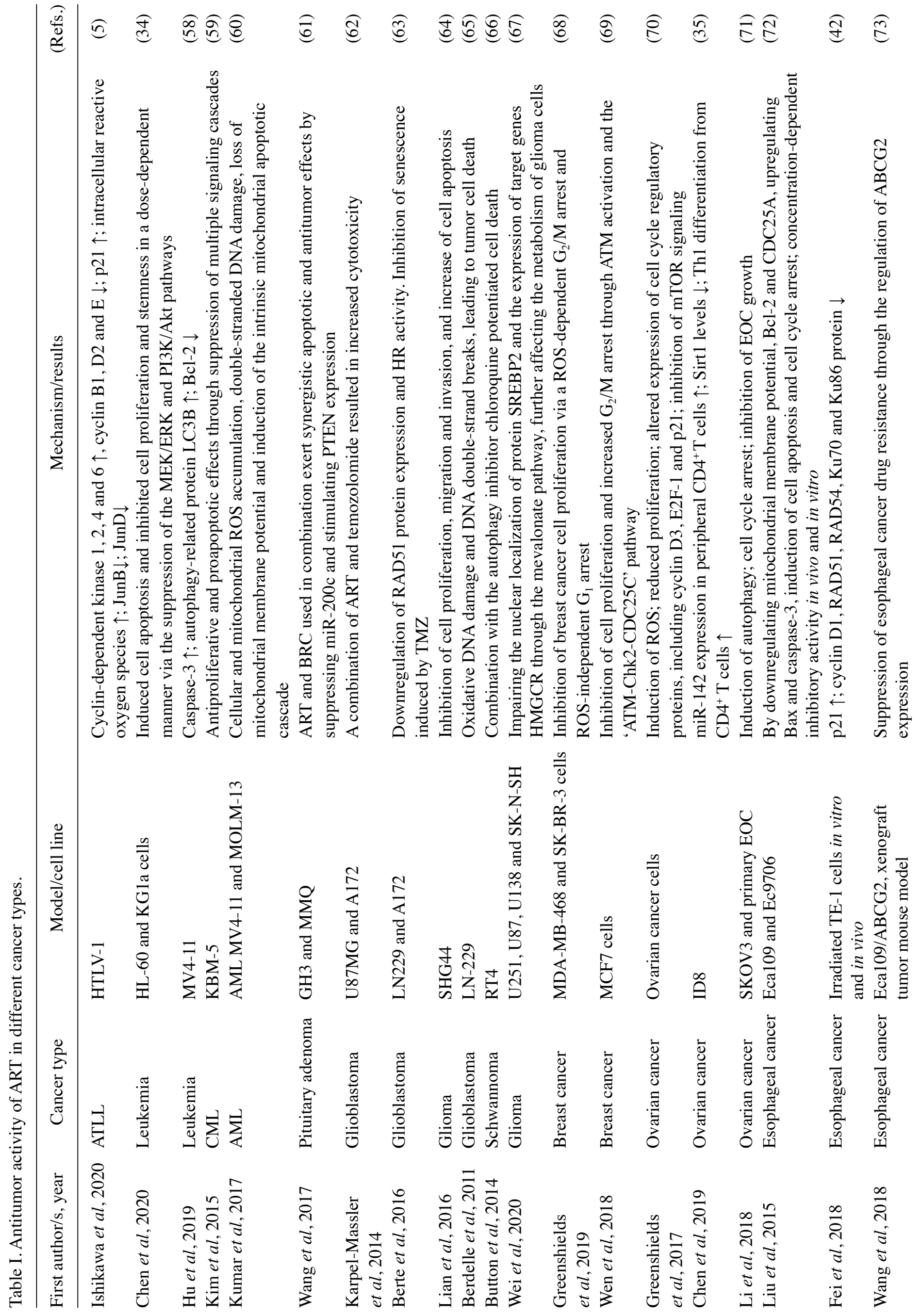




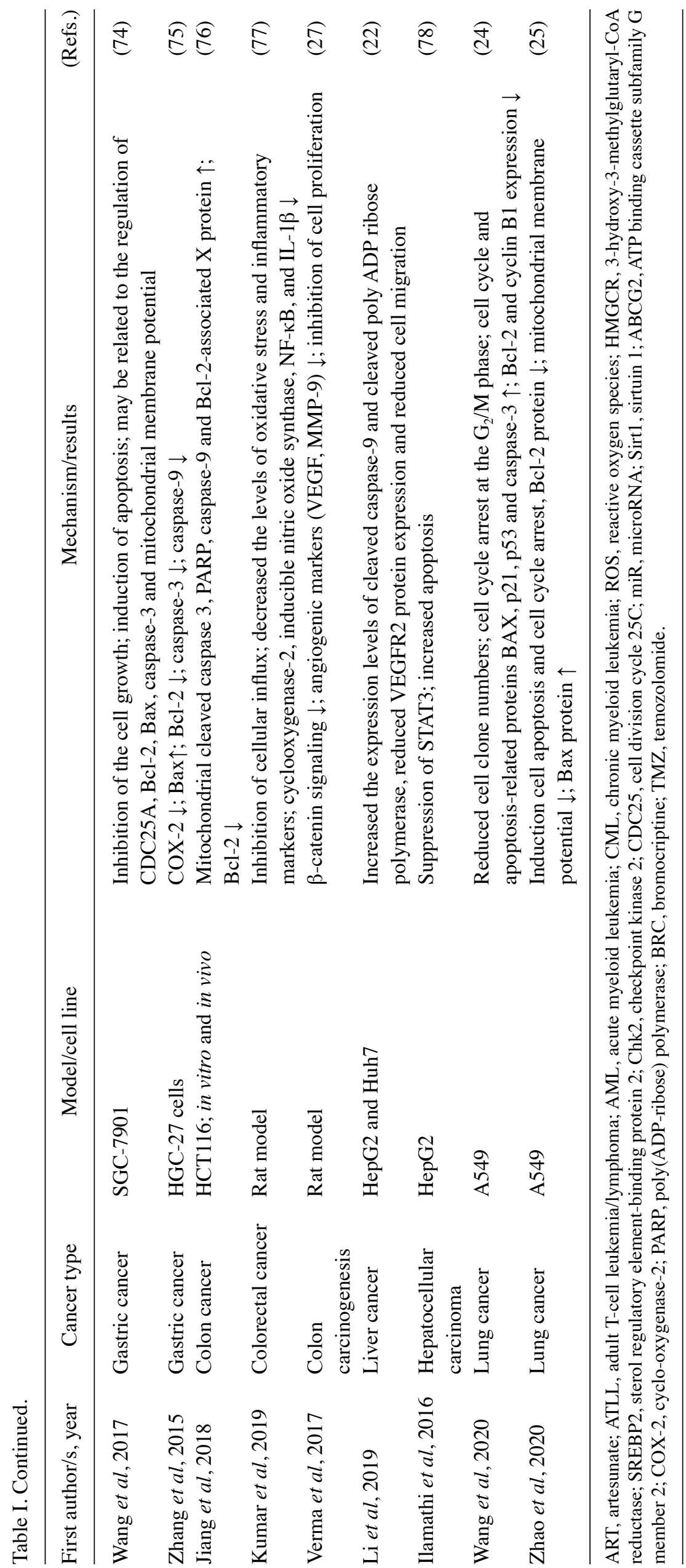


of survivin and induce the DNA damage response in glial cells to increase cell apoptosis and cell cycle arrest, resulting in increased sensitivity to radiotherapy (88). Previously, Wei et al (67) found that ART affected the nuclear localization of sterol regulatory element-binding protein 2 (SREBP2) by decreasing the expression of 3-hydroxy-3-methylglutaryl-CoA reductase and inhibited the mevalonate pathway, which in turn influenced the metabolism of glioma cells. In addition, ART disrupted the interaction between P53 and SREBP2 (which negatively regulates P53 and inhibits senescence), upregulated the expression of P21 and induced senescence in the U251, U87, U138 and SK-N-SH human glioma cell lines (67). The combination of ART and rapamycin (a specific inhibitor of mTOR) has been shown to synergistically decrease translation-controlled tumor protein (TCTP) expression and enhance the cytotoxicity of malignant peripheral nerve sheath tumor (MPNST) cells via mTOR-TCTP positive feedback loop, the results also suggested that TCTP may be a new target for the treatment of neurofibromatosis type 1-associated tumors and MPNSTs (89).

Thyroid carcinoma. Thyroid cancer is the most common cancer in the endocrine system, and its incidence is increasing worldwide (90). Anaplastic thyroid carcinoma (ATC) is an aggressive malignancy that is almost always fatal and lacks effective systemic treatment options. It is highly resistant to chemotherapy due to its undifferentiated and aggressive characteristics $(91,92)$. Ma and Fei (91) showed that ART could inhibit growth and induce apoptosis in ATC cells $(8505 \mathrm{C}$, 8505C-r, KAT-4-r and KAT-4), by suppressing mitochondrial respiration and acting synergistically with chemotherapy drug doxorubicin without affecting glycolysis. Thus, ART led to oxidative stress and damage in ATC cells. Their work suggested that ART was a potential complement to the treatment of ATC, particularly cases with chemoresistance (91).

Breast cancer. Breast cancer is one of the most common malignancies among women (93). Despite decades of laboratory, epidemiological and clinical research, breast cancer rates continue to rise. Breast cancer remains the leading cancer-related cause of the burden of disease among women, affecting 1 in 20 women globally and 1 in 8 women in high-income countries $(94,95)$. Systemic treatment (chemotherapy and endocrine therapy) of breast cancer is initially effective; however, after a period of time, drug resistance typically develops (96). ART has also been found to block the cell cycle progression of MCF-7 and MDA-MB-231 cells at the $G_{2} / M$ phase and upregulate the expression of p21 and Beclin-1, thereby inhibiting the proliferation of breast cancer cells by inducing autophagy (53). ART treatment was also revealed to inhibit the proliferation of the triple-negative breast cancer cell line MDA-MB-468 and the human epidermal growth factor-2-enriched breast cancer cell line SK-BR-3 in a dose- and time-dependent manner. The proliferation of MDA-MB-468 and SK-BR-3 cells was inhibited by ROS-dependent $\mathrm{G}_{2} / \mathrm{M}$ cell cycle arrest and ROS-independent $\mathrm{G}_{1}$ cell cycle arrest (68). Furthermore, ART can inhibit breast cancer MCF-7 cell proliferation and promote $G_{2} / M$ arrest by activating the ataxia-telangiectasia mutated/checkpoint kinase 2/cell division cycle 25 (CDC25) C pathway (69). By loading ART into the lipid core of a polymer-lipid hybrid carrier, the anticancer activity and physical stability of ART were found to be significantly increased and can be used for chemotherapy (97-99). Raza et al (100) found that ART generated the reactive oxygen species (ROS), resulted in DNA damage and enhanced the apoptosis of neighboring cells (Cx43-MCF7 cells) in breast cancer MCF-7 cells. In addition, the dose-dependent cytotoxicity of ART could be reduced by the gap junction (GJ) protein connexin-43 (Cx43). Li et al (101) found that ART could inhibit lysosomal function and clear dysfunctional mitochondria, and induce breast cancer cell apoptosis. In addition, ART was found to have a stronger inhibitory effect on drug-resistant breast cancer cells (A549/TAX and MCF-7/ADR) with higher lysosomal functional activity (101).

Ovarian cancer. Ovarian cancer is the seventh most common type of malignant neoplasm in women and the eighth cause of mortality (102-104). Most patients with ovarian cancer are typically diagnosed at an advanced stage of the disease (105). Ovarian cancer is treated with platinum chemotherapy following surgical resection (106). However, the recurrence rate is high $(107,108)$ and the survival rates of ovarian cancer with International Federation of Gynecology and Obstetrics stage III and IV are only 10-30\% (109). ART has been found to significantly reduce the expression of VEGF in the HO-8910 human ovarian cancer cell line, as well as that of KDR/flk-1 (VEGF receptor) in endothelial cells and HO-8910 cells, thereby significantly inhibiting angiogenesis in a dose-dependent form. Additionally, ART resulted in reduced xenograft tumor growth in nude mice, with no clear toxicity to the animal (110). ART could reduce the total amount of RAD51 and the formation of RAD51 foci in ovarian cancer cells sustaining DNA damage. Moreover, the downregulation of RAD51 conferred ovarian cancer cells an increased sensitivity to cisplatin (111). ART combined with cisplatin can synergistically induce DNA double-strand breaks and inhibit the proliferation of the HO8910 and SKOV-3 human ovarian cancer cell lines (111). ART induced the production of ROS and reduced proliferation in HEY1, HEY2 and SKOV-3 ovarian cancer cells, which were associated with downregulation in the expression levels of regulatory proteins of the cell cycle, including cyclin D3, CDKs (CDK4, CDK2, and CDK1), Rb, E2F-1 and CDC25C, while the tumor suppressor p21WAF1/CIP1, as well as phosphorylated Chk2 kinase which is important in the DNA damage response and an inhibitor of the CDC25 phosphatases were upregulated (70).

Esophageal cancer. Esophageal cancer (EC) is a common malignancy and has a high incidence rate in China (112). Although therapeutic approaches have improved, the 5-year survival of EC is $<20 \%$ (113). ART can induce apoptosis and cell cycle arrest in the Eca109 and Ec9706 EC cell lines by upregulating Bax and caspase- 3 and reducing mitochondrial membrane potential, as well as Bcl-2 and CDC25A expression in a concentration-dependent manner (72). In addition, an in vivo study showed that ART produced a dose-dependent Eca109-transplanted tumor regression in Balb/c nude mice, with little side effects. These results revealed that CDC25A was a molecular target of ART and that ART could inhibit the growth of EC cells by inducing apoptosis and $\mathrm{G}_{0} / \mathrm{G}_{1}$ cell cycle 
arrest (72). Fei et al (42) demonstrated that ART inhibited the proliferation of EC cells, enhanced radiosensitivity of TE-1 cells in vitro and enhanced the effect of apoptosis induced by irradiation in TE-1 cells by upregulating P21 and downregulating the expression of cyclin D1, RAD51, RAD54, $\mathrm{Ku} 70$ and Ku86 protein of irradiated TE- 1 cells. Moreover, ART also could aggravate DNA damage of EC cells and prolong the formation of $\gamma-\mathrm{H} 2 \mathrm{AX}$ foci induced by IR in TE- 1 cells. The results indicated that $\mathrm{ART}$ may be a promising radiosensitizer for the treatment of EC. In another study, Wang et al (73) found that ART can reverse doxorubicin resistance in EC by downregulating the expression of ATP-binding cassette G2 in Eca109 cells. ART was reported to inhibit the proliferation, migration and invasion of KYSE-150 esophageal squamous cell carcinoma cells by suppressing cell elasticity and increasing adhesion; ART also may increase the apoptosis rate by altering the cytoskeleton of KYSE-150 cells (114).

Gastric cancer. Gastric cancer is the fourth leading cause of cancer-related mortality in the world, with a 5-year survival rate of $<40 \%(115,116)$. ART can inhibit the proliferation of the gastric cancer cell lines SGC-7901, BGC-823 and AGS in a concentration-dependent manner, BGC-823 cells treated with ART exhibited calcium overload, downregulated expression levels of VEGF and upregulated expression levels of calpain-2 (117). ART treatment can also inhibit the proliferation of the SGC-7901 gastric adenocarcinoma cell line and induce apoptosis; the mechanism may be associated with Bax and caspase-3 upregulation and CDC25A and Bcl-2 downregulation (74). In addition, ART could prevent the growth of Helicobacter pylori and gastric cancer cells, inhibit the adhesion of Helicobacter pylori to these cells and reduce Helicobacter pylori-enhanced ROS production. Moreover, ART significantly reduces the number of tumor nodules and tumor size in a gastric cancer mouse model by inhibiting the $\mathrm{NF}-\kappa \mathrm{B}$ signaling pathway (118).

Colorectal cancer. Colorectal cancer (CRC) is one of the most common types of cancer worldwide and has incidence and mortality rates globally $(119,120)$. ART was found to inhibit CRC proliferation and promote apoptosis in a dose-dependent manner to significantly suppress the growth of colorectal tumors, decrease the physiological activity of cancer and delay spontaneous liver metastasis in the CLY CRC cell line. These anticancer effects were associated with the membrane translocation of $\beta$-catenin and the inhibition of unrestricted Wnt/ $\beta$-catenin signaling (121). In addition, ART can reverse the immunosuppression by downregulating the concentrations of TGF- $\beta 1$ and IL-10 in Colon26 and RKO CRC cells (122). Jiang et al (76) found that ART induced apoptosis by increasing the protein levels of cleaved caspase-3, poly-ADP ribose polymerase (PARP), caspase- 9 and Bax protein levels, while decreasing the levels of LC3 and beclin-1 in HCT116 colon cancer cells. ART can reduce the levels of oxidative stress and inflammatory markers, downregulate cyclo-oxygenase-2, induce nitric oxide (NO) synthase, NF- $\kappa$ B and IF-1 $\beta$ and reduce the risk of colon cancer (77).

Lung cancer. Lung cancer is the most common cancer in the world and the leading cause of cancer death (123), which has an overall 5-year survival rate of $\sim 15 \%$ (124). Despite advances in treatment, progressive NSCLC still severely limits survival and requires new therapeutic compounds (125). ART can significantly inhibit the invasion and migration of NSCLC cells (H1395, A549, LXF289 and H460 cells) by downregulating the transcription of urokinase-type plasminogen activator, MMP-2 and MMP-7, whilst inhibiting AP-1 and NF- $\kappa$ B-transactivation (126). In addition, ART promotes radiosensitivity in A549 cells in vitro and in vivo, possibly by inducing cell cycle arrest at the $\mathrm{G}_{2} / \mathrm{M}$ phase through the NO signaling pathway (127). Wang et al (128) found that ART could inhibit cell migration by upregulating the expression of the epithelial marker E-cadherin in A549 and H1975 NSCLC cell lines. In another study, ART could inhibit the invasion of A549 cells, and the mechanism may be associated with the reduced expression of intercellular adhesion molecule-1 and MMP-9 (129). Furthermore, ART inhibits the proliferation of A549 and H1299 cells by arresting the cell cycle at the $G_{1}$ phase and suppresses lung tumor progression by inhibiting the $\mathrm{Wnt} / \beta$-catenin pathway (130). In A549 cells, ART combined with cisplatin blocks the cell cycle at the $G_{2} / M$ phase and induces apoptosis by upregulating the expression of Bax, p53, p21, caspase-3, caspase-7 and caspase-9, whilst synergistically regulating the activity of the MAPK pathway by downregulating p-P38, p-JNK and p-ERK levels, which results in potentiated effects against cancer cell proliferation on A549 cells (131).

Liver cancer. Liver cancer is highly malignant and insensitive to cytotoxic chemotherapy, and is associated with a very poor patient prognosis $(132,133)$. ART can activate caspase-3, increase the $\mathrm{Bax} / \mathrm{Bcl}-2$ ratio and PARP, whilst downregulating mouse double minute 2, which leads to induced apoptosis on human hepatocellular carcinoma (HCC) cells but had little effect on normal cells (134). The anticancer effects of ART nanoliposomes on human HepG2 cells was stronger than those mediated by ART active pharmaceutical ingredient at the same concentration (135). ART may function as a potential inhibitor of STAT3 in HCC cells to regulate STAT3 targets, including caspase-3, Bcl-xl and survivin, interfere with STAT3 dimerization and inhibition of both constitutive and IL-6-inducible STAT3, leading to cell apoptosis in vitro (78). Jing et al (30) also revealed that ART could inhibit phosphorylation of AKT and mTOR significantly, and induce apoptosis in HCC (SK-hep1 and SM-7721 cell lines) by inhibiting the PI3K/AKT/mTOR pathway. In addition, ART combined with sorafenib (which is a novel multi-targeted oral drug for the treatment of cancer) further increased the apoptosis of HCC cells by dual inhibition of both RAF/RAF/MAPK pathway and PI3K/AKT/mTOR pathway. Thus, the study identified a potential treatment strategy combining ART with sorafenib for the treatment of advanced HCC.

Other tumors. In a previous study, ART has been reported to induce lactate dehydrogenase release and cell death in necrosis-sensitive cholangiocarcinoma (136). Wang et al (137) found that ART could significantly inhibit proliferation in the Burkitt lymphoma Raji cell line, where it induced apoptosis and autophagy. The combination of ART and bromocriptine can synergistically promote apoptosis by inhibiting miR-200c expression and increasing that of PTEN in lactinomas (61). 
Chauhan et al (138) found that ART induced ROS production and subsequent cell death in a receptor-interacting protein 1-dependent manner in human renal carcinoma. ART exerted a potent antiproliferative effect on polyomavirus-positive Merkel cell carcinoma (MCC) cells with good overall tolerance and induced ferroptosis (139). In addition, ART also significantly suppressed the growth of established MCC tumors in xenotransplanted mice, suggesting that ART may be used for the treatment of MCC (138). In another study, ART blocked the Wnt/catenin pathway to inhibit the proliferation, migration and invasion of uveal melanoma cells (primary 92.1 and metastatic Omm2.3 UM cells), mainly by suppressing the phosphorylation of GSK $3 \beta$ at Ser9 and decreasing the protein levels of $\beta$-catenin and its downstream targets (c-Myc and cyclin D1) (140). Wang et al (141) found that ART decreased androgen receptor (AR) expression, increased the expression and the catalytic activity of DNA methyltransferase3b (DNMT3b) in 22rv1 cells either in transplanted mice or in vitro. ART can suppress tumor growth of prostatic cancer cells through AR-DNMT3b pathway, suggesting it may be used for the treatment of prostate cancer in the future. Yang et al (142) found that ART induced mitochondrial dysfunction and cell apoptosis in the WERI-Rb1 and Y79 human retinoblastoma cell lines and in the ARPE-19 human retinal pigment epithelium cell line by upregulating Kruppel-like factor 6 expression, increasing the $\mathrm{Bax} / \mathrm{Bcl}-2$ ratio, promoting the release of cytochrome $c$ and stimulating the cleavage of caspase- 9 and -3 . Roh et al (143) demonstrated that ART could induce ferroptosis in head and neck (HNC) cells via cellular glutathione depletion and ROS accumulation, and ART sensitivity decreased in some cisplatin-resistant HNCs as a result of Nrf2-ARE pathway activation. Berköz et al (144) suggested that ART treatment could decrease cell migration, invasion and colony formation in the A375 human melanoma cell line, possibly by inhibiting STAT3, Src activation and the protein expression of STAT3-associated molecules, including MMP-2, MMP-9, myeloid-cell leukemia 11, Bcl-xl, VEGF and Twist.

\section{Summary and perspectives}

Cancer is one of the most life-threatening diseases. With the increasing prevalence of cancer, the development of anticancer agents has become a key field of clinical and scientific research. Developments in medical science and technology have enabled the extraction of bioactive components from Traditional Chinese medicines for research due to their reported anticancer effects and lack of adverse reactions. ART has been demonstrated to be effective against leukemia, breast cancer, gastrointestinal tumors and other types of cancer $(8,23,145)$. Importantly, since it is a drug that is already being used for the treatment of malaria, ART has a reliable safety record for clinical use. Although the amount of clinical data regarding the use of ART as an anticancer drug remains limited, preliminary results have been encouraging in terms of efficacy and tolerance (22). Combination therapy should be a key consideration in the future. In addition, development of modified derivatives of ART after structural modifications or modifying the treatment regimen to optimize the efficacy and toxicity profile are also possible directions for future research.
To conclude, existing information provides evidence supporting the use of ART as an anticancer agent. However, data from systematic in vivo animal and human studies are required to improve our understanding of the anticancer effects and mechanism of action of ART in the future.

\section{Acknowledgements}

Not applicable.

\section{Funding}

The present study was supported by the Health Commission of Hubei Province scientific research project (grant no. WJ2021Q015), the College Students Innovation Program of Yangtze University (grant no. Yz2020338) and the Health Commission of Hubei Province Scientific Research Project (grant no. WJ2019-17).

\section{Availability of data and materials}

Data sharing is not applicable to this article, as no datasets were generated or analyzed during the present study.

\section{Authors' contributions}

LL designed and supervised the study. JH, YZ and FW reviewed the references. $\mathrm{XY}$ wrote the manuscript. YZ, FW and $\mathrm{JZ}$ contributed to the table and figure. $\mathrm{XY}$ and LL revised the manuscript. All authors read and approved the final manuscript. Data authentication is not applicable.

\section{Ethics approval and consent to participate}

Not applicable.

\section{Patient consent for publication}

Not applicable.

\section{Competing of interests}

The authors declare that they have no competing interests.

\section{References}

1. Miller KD, Fidler-Benaoudia M, Keegan TH, Hipp HS, Jemal A and Siegel RL: Cancer statistics for adolescents and young adults, 2020. CA Cancer J Clin 70: 443-459, 2020.

2. Siegel RL, Miller KD, Fuchs HE and Jemal A: Cancer statistics, 2021. CA Cancer J Clin 71: 7-33, 2021.

3. Cao W, Chen HD, Yu YW, Li N and Chen WQ: Changing profiles of cancer burden worldwide and in China: A secondary analysis of the global cancer statistics 2020. Chin Med J (Engl) 134: 783-791, 2021.

4. Sung H, Ferlay J, Siegel RL, Laversanne M, Soerjomataram I, Jemal A and Bray F: Global cancer statistics 2020: GLOBOCAN estimates of incidence and mortality worldwide for 36 cancers in 185 countries. CA Cancer J Clin 71: 209-249, 2021.

5. Ishikawa C, Senba M and Mori N: Evaluation of artesunate for the treatment of adult T-cell leukemia/lymphoma. Eur J Pharmacol 872: 172953, 2020.

6. Slezakova S andf Ruda-Kucerova J: Anticancer activity of artemisinin and its derivatives. Anticancer Res 37: 5995-6003, 2017. 
7. Cen YY, Zao YB, Li P, Li XL, Zeng XX and Zhou H: Research progress on pharmacokinetics and pharmacological activities of artesunate. Zhongguo Zhong Yao Za Zhi 43: 3970-3978, 2018 (In Chinese)

8. Khanal P: Antimalarial and anticancer properties of artesunate and other artemisinins: Current development. Monatsh Chem: Mar 30, 2021 (Epub ahead of print)

9. Zhou X, Chen Y, Wang F, Wu H, Zhang Y, Liu J, Cai Y, Huang S, He N, Hu Z and Jin X: Artesunate induces autophagy dependent apoptosis through upregulating ROS and activating AMPK-mTOR-ULK1 axis in human bladder cancer cells. Chem Biol Interact 331: 109273, 2020.

10. Zhao F, Vakhrusheva O, Markowitsch SD, Slade KS, Tsaur I, Cinatl J Jr, Michaelis M, Efferth T, Haferkamp A and Juengel E: Artesunate impairs growth in cisplatin-resistant bladder cancer cells by cell cycle arrest, apoptosis and autophagy induction. Cells 9: 2643, 2020.

11. Markowitsch SD, Schupp P, Lauckner J, Vakhrusheva O, Slade KS, Mager R, Efferth T, Haferkamp A and Juengel E: Artesunate inhibits growth of sunitinib-resistant renal cell carcinoma cells through cell cycle arrest and induction of ferroptosis. Cancers (Basel) 12: 3150, 2020.

12. Klaunig JE: Oxidative stress and cancer. Curr Pharm Des 24 $4771-4778,2018$

13. Lichota A and Gwozdzinski K: Anticancer activity of natural compounds from plant and marine environment. Int $\mathbf{J}$ Mol Sci 19: 3533, 2018

14. Chekem L and Wierucki S: Extraction of artemisinin and synthesis of its derivates artesunate and artemether. Med Trop (Mars) 66: 602-605, 2006 (In French)

15. Efferth T: From ancient herb to modern drug: Artemisia annua and artemisinin for cancer therapy. Semin Cancer Biol 46: 65-83, 2017.

16. Wei $\mathrm{T}$ and Liu J: Anti-angiogenic properties of artemisinin derivatives (review). Int J Mol Med 40: 972-978, 2017.

17. Ho WE, Peh HY, Chan TK and Wong WS: Artemisinins: Pharmacological actions beyond anti-malarial. Pharmacol Ther 142: 126-139, 2014.

18. Zhang J, Sun X, Wang L, Wong YK, Lee YM, Zhou C, Wu G, Zhao T, Yang L, Lu L, et al: Artesunate-induced mitophagy alters cellular redox status. Redox Biol 19: 263-273, 2018.

19. Alagbonsi AI, Salman TM, Sulaiman SO, Adedini KA and Kebu S: Possible mechanisms of the hypoglycaemic effect of artesunate: Gender implication. Metabol Open 10: 100087 2021.

20. Venturini E, Zammarchi L, Bianchi L, Montagnani C, Tersigni C Bortone B, Chiappini E and Galli L: Efficacy and safety of intravenous artesunate in children with severe imported malaria. Pediatr Infect Dis J 39: e220, 2020.

21. Li Z, Shi X, Liu J, Shao F, Huang G, Zhou Z and Zheng P: Artesunate prevents type 1 diabetes in NOD mice mainly by inducing protective IL-4-producing $\mathrm{T}$ cells and regulatory $\mathrm{T}$ cells. FASEB J 33: 8241-8248, 2019.

22. $\mathrm{Li} \mathrm{H}, \mathrm{Xu} \mathrm{K}$, Pian G and Sun S: Artesunate and sorafenib: Combinatorial inhibition of liver cancer cell growth. Oncol Lett 18: 4735-4743, 2019

23. Pirali M,Taheri M,Zarei S, Majidi M and Ghafouri H: Artesunate, as a HSP70 ATPase activity inhibitor, induces apoptosis in breast cancer cells. Int J Biol Macromol 164: 3369-3375, 2020.

24. Wang Z, Wang Q, He T, Li W, Liu Y, Fan Y, Wang Y, Wang Q and Chen J: The combination of artesunate and carboplatin exerts a synergistic anti-tumour effect on non-small cell lung cancer. Clin Exp Pharmacol Physiol 47: 1083-1091, 2020.

25. Zhao Y, Liu J and Liu L: Artesunate inhibits lung cancer cells via regulation of mitochondrial membrane potential and induction of apoptosis. Mol Med Rep 22: 3017-3022, 2020.

26. Zhang Q, Yi H, Yao H, Lu L, He G, Wu M, Zheng C, Li Y, Chen S, Li L, et al: Artemisinin derivatives inhibit non-smal cell lung cancer cells through induction of ROS-dependent apoptosis/ferroptosis. J Cancer 12: 4075-4085, 2021.

27. Verma S, Das P and Kumar VL: Chemoprevention by artesunate in a preclinical model of colorectal cancer involves down regulation of $\beta$-catenin, suppression of angiogenesis, cellular proliferation and induction of apoptosis. Chem Biol Interact 278: 84-91, 2017.

28. Ma JD, Jing J, Wang JW, Yan T, Li QH, Mo YQ, Zheng DH, Gao JL, Nguyen KA and Dai L: A novel function of artesunate on inhibiting migration and invasion of fibroblast-like synoviocytes from rheumatoid arthritis patients. Arthritis Res Ther 21: 153, 2019.
29. Qian P, Zhang YW, Zhou ZH, Liu JQ, Yue SY, Guo XL, Sun LQ Lv XT and Chen JQ: Artesunate enhances $\gamma \delta$ T-cell-mediated antitumor activity through augmenting $\gamma \delta$ T-cell function and reversing immune escape of HepG2 cells. Immunopharmacol Immunotoxicol 40: 107-116, 2018.

30. Jing W, Shuo L, Yingru X, Min M, Runpeng Z, Jun X and Dong H: Artesunate promotes sensitivity to sorafenib in hepatocellular carcinoma. Biochem Biophys Res Commun 519: 41-45, 2019

31. He W, Huang X, Berges BK, Wang Y, An N, Su R and Lu Y: Artesunate regulates neurite outgrowth inhibitor protein $B$ receptor to overcome resistance to sorafenib in hepatocellular carcinoma cells. Front Pharmacol 12: 615889, 2021.

32. Xu X, Lai Y and Hua ZC: Apoptosis and apoptotic body: Disease message and therapeutic target potentials. Biosci Rep 39: BSR20180992, 2019.

33. Wong YK, Xu C, Kalesh KA, He Y, Lin Q, Wong WSF, Shen HM and Wang J: Artemisinin as an anticancer drug: Recent advances in target profiling and mechanisms of action. Med Res Rev 37: 1492-1517, 2017.

34. Chen S, Gan S, Han L, Li X, Xie X, Zou D and Sun H: Artesunate induces apoptosis and inhibits the proliferation, stemness, and tumorigenesis of leukemia. Ann Transl Med 8: 767, 2020.

35. Chen X,Zhang XL,Zhang GH and Gao YF: Artesunate promotes Th1 differentiation from CD4+ T cells to enhance cell apoptosis in ovarian cancer via miR-142. Braz J Med Biol Res 52: e7992, 2019.

36. Peng J, Yuan C, Wu Z, Wang Y, Yin W, Lin Y, Zhou L and Lu J: Upregulation of microRNA-1 inhibits proliferation and metastasis of breast cancer. Mol Med Rep 22: 454-464, 2020.

37. Zhang J, Zhou L, Xiang JD, Jin CS, Li MQ and He YY: Artesunate-induced ATG5-related autophagy enhances the cytotoxicity of NK92 cells on endometrial cancer cells via interactions between CD155 and CD226/TIGIT. Int Immunopharmacol 97: 107705, 2021.

38. Hayes JD, Dinkova-Kostova AT and Tew KD: Oxidative stress in cancer. Cancer Cell 38: 167-197, 2020.

39. Kohan R, Collin A, Guizzardi S, Tolosa de Talamoni N and Picotto G: Reactive oxygen species in cancer: A paradox between pro- and anti-tumour activities. Cancer Chemother Pharmacol 86: 1-13, 2020.

40. Kirtonia A, Sethi G and Garg M: The multifaceted role of reactive oxygen species in tumorigenesis. Cell Mol Life Sci 77: 4459-4483, 2020.

41. Srinivas US, Tan BWQ, Vellayappan BA and Jeyasekharan AD: ROS and the DNA damage response in cancer. Redox Biol 25: 101084, 2019

42. Fei Z, Gu W, Xie R, Su H and Jiang Y: Artesunate enhances radiosensitivity of esophageal cancer cells by inhibiting the repair of DNA damage. J Pharmacol Sci 138: 131-137, 2018

43. Petricciuolo M, Davidescu M, Fettucciari K, Gatticchi L, Brancorsini S, Roberti R, Corazzi L and Macchioni L: The efficacy of the anticancer 3-bromopyruvate is potentiated by antimycin and menadione by unbalancing mitochondrial ROS production and disposal in U118 glioblastoma cells. Heliyon 6: e05741, 2020

44. Yu X, Wang X, Wang X, Zhou Y, Li Y, Wang A, Wang T, An Y, Sun W, Du J, et al: TEOA inhibits proliferation and induces DNA damage of diffuse large b-cell lymphoma cells through activation of the ROS-dependent p38 MAPK signaling pathway. Front Pharmacol 11: 554736, 2020.

45. Zhang H, Li M, Zhu X, Zhang Z, Huang H and Hou L: Artemisinin co-delivery system based on manganese oxide for precise diagnosis and treatment of breast cancer. Nanotechnology: Apr 28, 2021 (Epub ahead of print).

46. Yao X, Zhao CR, Yin H, Wang K and Gao JJ: Synergistic antitumor activity of sorafenib and artesunate in hepatocellular carcinoma cells. Acta Pharmacol Sin 41: 1609-1620, 2020.

47. Viallard $C$ and Larrivée B: Tumor angiogenesis and vascular normalization: Alternative therapeutic targets. Angiogenesis 20: 409-426, 2017

48. Li S, Xu HX, Wu CT, Wang WQ, Jin W, Gao HL, Li H, Zhang SR, $\mathrm{Xu}$ JZ, Qi ZH, et al: Angiogenesis in pancreatic cancer: Current research status and clinical implications. Angiogenesis 22: 15-36, 2019.

49. Cao J, Liu X, Yang Y, Wei B, Li Q, Mao G, He Y, Li Y, Zheng L, Zhang $\mathrm{Q}$, et al: Decylubiquinone suppresses breast cancer growth and metastasis by inhibiting angiogenesis via the ROS/p53/BAI1 signaling pathway. Angiogenesis 23: 325-338, 2020. 
50. Singh N, Badrun D and Ghatage P: State of the art and up-and-coming angiogenesis inhibitors for ovarian cancer. Expert Opin Pharmacother 21: 1579-1590, 2020.

51. Chen H, Shi L, Yang X, Li S, Guo X and Pan L: Artesunate inhibiting angiogenesis induced by human myeloma RPMI8226 cells. Int J Hematol 92: 587-597, 2010.

52. Andrade-Tomaz M, de Souza I, Rocha CRR and Gomes LR: The role of chaperone-mediated autophagy in cell cycle control and its implications in cancer. Cells 9: 2140, 2020.

53. Chen K, Shou LM, Lin F, Duan WM, Wu MY, Xie X, Xie YF, $\mathrm{Li} \mathrm{W}$ and Tao M: Artesunate induces G2/M cell cycle arrest through autophagy induction in breast cancer cells. Anticancer Drugs 25: 652-662, 2014

54. Weng X, Zhu SQ and Cui HJ: Artesunate inhibits proliferation of glioblastoma cells by arresting cell cycle. Zhongguo Zhong Yao Za Zhi 43: 772-778, 2018 (In Chinese).

55. Wang Y, Wei Z, Pan K, Li J and Chen Q: The function and mechanism of ferroptosis in cancer. Apoptosis 25: 786-798, 2020

56. Liu Z, Zhao Q, Zuo ZX, Yuan SQ, Yu K, Zhang Q, Zhang X, Sheng H, Ju HQ, Cheng H, et al: Systematic analysis of the aberrances and functional implications of ferroptosis in cancer. iScience 23: 101302, 2020.

57. Li ZJ, Dai HQ, Huang XW, Feng J, Deng JH, Wang ZX, Yang XM, Liu YJ, Wu Y, Chen PH, et al: Artesunate synergizes with sorafenib to induce ferroptosis in hepatocellular carcinoma. Acta Pharmacol Sin 42: 301-310, 2021.

58. Hu LJ, Jiang T, Wang FJ, Huang SH, Cheng XM and Jia YQ Effects of artesunate combined with bortezomib on apoptosis and autophagy of acute myeloid leukemia cells in vitro and its mechanism. Zhonghua Xue Ye Xue Za Zhi 40: 204-208, 2019 (In Chinese).

59. Kim C, Lee JH, Kim SH, Sethi G and Ahn KS: Artesunate suppresses tumor growth and induces apoptosis through the modulation of multiple oncogenic cascades in a chronic myeloid leukemia xenograft mouse model. Oncotarget 6: 4020-4035, 2015.

60. Kumar B, Kalvala A, Chu S, Rosen S, Forman SJ, Marcucci G, Chen CC and Pullarkat V: Antileukemic activity and cellular effects of the antimalarial agent artesunate in acute myeloid leukemia. Leuk Res 59: 124-135, 2017.

61. Wang X, Du Q, Mao Z, Fan X, Hu B, Wang Z, Chen Z, Jiang X, Wang Z, Lei N, et al: Combined treatment with artesunate and bromocriptine has synergistic anticancer effects in pituitary adenoma cell lines. Oncotarget 8: 45874-45887, 2017.

62. Karpel-Massler G, Westhoff MA, Kast RE, Dwucet A, Nonnenmacher L, Wirtz CR, Debatin KM and Halatsch ME: Artesunate enhances the antiproliferative effect of temozolomide on U87MG and A172 glioblastoma cell lines. Anticancer Agents Med Chem 14: 313-318, 2014

63. Berte N, Lokan S, Eich M, Kim E and Kaina B: Artesunate enhances the therapeutic response of glioma cells to temozolomide by inhibition of homologous recombination and senescence. Oncotarget 7: 67235-67250, 2016.

64. Lian S, Shi R, Huang X, Hu X, Song B, Bai Y, Yang B, Dong J, $\mathrm{Du} \mathrm{Z}$, Zhang Y, et al: Artesunate attenuates glioma proliferation, migration and invasion by affecting cellular mechanical properties. Oncol Rep 36: 984-990, 2016.

65. Berdelle N, Nikolova T, Quiros S, Efferth T and Kaina B Artesunate induces oxidative DNA damage, sustained DNA double-strand breaks, and the ATM/ATR damage response in cancer cells. Mol Cancer Ther 10: 2224-2233, 2011.

66. Button RW, Lin F, Ercolano E, Vincent JH, Hu B, Hanemann CO and Luo S: Artesunate induces necrotic cell death in schwannoma cells. Cell Death Dis 5: e1466, 2014.

67. Wei S, Liu L, Chen Z, Yin W, Liu Y, Ouyang Q, Zeng F, Nie Y and Chen T: Artesunate inhibits the mevalonate pathway and promotes glioma cell senescence. J Cell Mol Med 24: 276-284, 2020 .

68. Greenshields AL, Fernando W and Hoskin DW: The anti-malarial drug artesunate causes cell cycle arrest and apoptosis of triple-negative MDA-MB-468 and HER2-enriched SK-BR-3 breast cancer cells. Exp Mol Pathol 107: 10-22, 2019.

69. Wen L, Liu L, Wen L, Yu T and Wei F: Artesunate promotes G2/M cell cycle arrest in MCF7 breast cancer cells through ATM activation. Breast Cancer 25: 681-686, 2018.

70. Greenshields AL, Shepherd TG and Hoskin DW: Contribution of reactive oxygen species to ovarian cancer cell growth arrest and killing by the anti-malarial drug artesunate. Mol Carcinog 56: 75-93, 2017.
71. Li B, Bu S, Sun J, Guo Y and Lai D: Artemisinin derivatives inhibit epithelial ovarian cancer cells via autophagy-mediated cell cycle arrest. Acta Biochim Biophys Sin (Shanghai) 50: $1227-1235,2018$

72. Liu L, Zuo LF, Zuo J and Wang J: Artesunate induces apoptosis and inhibits growth of Eca109 and Ec9706 human esophageal cancer cell lines in vitro and in vivo. Mol Med Rep 12: 1465-1472, 2015.

73. Wang L, Liu L, Chen Y, Du Y, Wang J and Liu J: Correlation between adenosine triphosphate (ATP)-binding cassette transporter G2 (ABCG2) and drug resistance of esophageal cancer and reversal of drug resistance by artesunate. Pathol Res Pract 214: 1467-1473, 2018.

74. Wang L, Liu L, Wang $J$ and Chen Y: Inhibitory effect of artesunate on growth and apoptosis of gastric cancer cells. Arch Med Res 48: 623-630, 2017.

75. Zhang P, Luo HS, Li M and Tan SY: Artesunate inhibits the growth and induces apoptosis of human gastric cancer cells by downregulating COX-2. Onco Targets Ther 8: 845-854, 2015.

76. Jiang F, Zhou JY, Zhang D, Liu MH and Chen YG: Artesunate induces apoptosis and autophagy in HCT116 colon cancer cells, and autophagy inhibition enhances the artesunate-induced apoptosis. Int J Mol Med 42: 1295-1304, 2018.

77. Kumar VL, Verma S and Das P: Artesunate suppresses inflammation and oxidative stress in a rat model of colorectal cancer. Drug Dev Res 80: 1089-1097, 2019.

78. Ilamathi M, Santhosh S and Sivaramakrishnan V: Artesunate as an anti-cancer agent targets stat-3 and favorably suppresses hepatocellular carcinoma. Curr Top Med Chem 16: 2453-2463, 2016.

79. Wojcicki AV, Kasowski MM, Sakamoto KM and Lacayo N: Metabolomics in acute myeloid leukemia. Mol Genet Metab 130: 230-238, 2020.

80. Efferth T, Giaisi M, Merling A, Krammer PH and Li-Weber M: Artesunate induces ROS-mediated apoptosis in doxorubicin-resistant T leukemia cells. PLoS One 2: e693, 2007.

81. Wang Y, Yang J, Chen L, Wang J, Wang Y, Luo J, Pan L and Zhang X: Artesunate induces apoptosis through caspase-dependent and -independent mitochondrial pathways in human myelodysplastic syndrome SKM-1 cells. Chem Biol Interact 219: 28-36, 2014.

82. Tan M, Rong Y, Su Q and Chen Y: Artesunate induces apoptosis via inhibition of STAT3 in THP-1 cells. Leuk Res 62: 98-103, 2017.

83. Li Y, Feng L, Li Y, Jiang W, Shan N and Wang X: Artesunate possesses anti-leukemia properties that can be enhanced by arsenic trioxide. Leuk Lymphoma 55: 1366-1372, 2014.

84. Papanikolaou X, Johnson S, Garg T, Tian E, Tytarenko R, Zhang Q, Stein C, Barlogie B, Epstein J and Heuck C: Artesunate overcomes drug resistance in multiple myeloma by inducing mitochondrial stress and non-caspase apoptosis. Oncotarget 5: 4118-4128, 2014.

85. Blessing MM and Alexandrescu S: Embryonal tumors of the central nervous system: An update. Surg Pathol Clin 13: 235-247, 2020.

86. Alegría-Loyola MA, Galnares-Olalde JA and Mercado M: Tumors of the central nervous system. Rev Med Inst Mex Seguro Soc 55: 330-340, 2017 (In Spanish).

87. Francis SS, Wang R, Enders C, Prado I, Wiemels JL, Ma X and Metayer C: Socioeconomic status and childhood central nervous system tumors in California. Cancer Causes Control 32: 27-39, 2021.

88. Reichert S, Reinboldt V, Hehlgans S, Efferth T, Rödel C and Rödel F: A radiosensitizing effect of artesunate in glioblastoma cells is associated with a diminished expression of the inhibitor of apoptosis protein survivin. Radiother Oncol 103: 394-401, 2012.

89. Kobayashi D, Hirayama M, Komohara Y, Mizuguchi S, Wilson Morifuji M, Ihn H, Takeya M, Kuramochi A and Araki N: Translationally controlled tumor protein is a novel biological target for neurofibromatosis type 1-associated tumors. J Biol Chem 289: 26314-26326, 2014

90. Zhao J, Wen J, Wang S, Yao J, Liao L and Dong J: Association between adipokines and thyroid carcinoma: A meta-analysis of case-control studies. BMC Cancer 20: 788, 2020.

91. Ma L and Fei H: Antimalarial drug artesunate is effective against chemoresistant anaplastic thyroid carcinoma via targeting mitochondrial metabolism. J Bioenerg Biomembr 52: 123-130, 2020.

92. Capdevila J, Wirth LJ, Ernst T, Ponce Aix S, Lin CC, Ramlau R, Butler MO, Delord JP, Gelderblom H, Ascierto PA, et al: PD-1 blockade in anaplastic thyroid carcinoma. J Clin Oncol 38: 2620-2627, 2020 
93. Breast Cancer Expert Committee of National Cancer Quality Control Center; Breast Cancer Expert Committee of China Anti-Cancer Association; Cancer Drug Clinical Research Committee of China Anti-Cancer Association: Guidelines for clinical diagnosis and treatment of advanced breast cancer in China (2020 edition). Zhonghua Zhong Liu Za Zhi 42: 781-797, 2020 (In Chinese).

94. Barzaman K, Karami J, Zarei Z, Hosseinzadeh A, Kazemi MH, Moradi-Kalbolandi S, Safari E and Farahmand L: Breast cancer: Biology, biomarkers, and treatments. Int Immunopharmacol 84 106535,2020

95. Britt KL, Cuzick J and Phillips KA: Key steps for effective breast cancer prevention. Nat Rev Cancer 20: 417-436, 2020

96. Dong X, Bai X, Ni J, Zhang H, Duan W, Graham P and Li Y: Exosomes and breast cancer drug resistance. Cell Death Dis 11: $987,2020$.

97. Tran TH, Nguyen TD, Poudel BK, Nguyen HT, Kim JO, Yong CS and Nguyen CN: Development and evaluation of artesunate-loaded chitosan-coated lipid nanocapsule as a potential drug delivery system against breast cancer. AAPS PharmSciTech 16: 1307-1316, 2015.

98. Tran TH, Nguyen AN, Kim JO, Yong CS and Nguyen CN: Enhancing activity of artesunate against breast cancer cells via induced-apoptosis pathway by loading into lipid carriers. Artif Cells Nanomed Biotechnol 44: 1979-1987, 2016.

99. Zhang S, Yuan H, Guo Y, Wang K, Wang X and Guo Z: Towards rational design of RAD51-targeting prodrugs: platinum ${ }^{\mathrm{IV}}$-artesunate conjugates with enhanced cytotoxicity against BRCA-proficient ovarian and breast cancer cells. Chem Commun (Camb) 54: 11717-11720, 2018

100. Raza A, Ghoshal A, Chockalingam S and Ghosh SS: Connexin-43 enhances tumor suppressing activity of artesunate via gap junction-dependent as well as independent pathways in human breast cancer cells. Sci Rep 7: 7580, 2017.

101. Li Z, Zhu YT, Xiang M, Qiu JL, Luo SQ and Lin F: Enhanced lysosomal function is critical for paclitaxel resistance in cancer cells: Reversed by artesunate. Acta Pharmacol Sin 42: 624-632, 2021.

102. Kujawa KA and Lisowska KM: Ovarian cancer-from biology to clinic. Postepy Hig Med Dosw (Online) 69: 1275-1290, 2015 (In Polish).

103. Aziz NB, Mahmudunnabi RG, Umer M, Sharma S, Rashid MA Alhamhoom Y, Shim YB, Salomon C and Shiddiky MJA: MicroRNAs in ovarian cancer and recent advances in the development of microRNA-based biosensors. Analyst 145 2038-2057, 2020

104. Gaona-Luviano P, Medina-Gaona LA and Magaña-Pérez K Epidemiology of ovarian cancer. Chin Clin Oncol 9: 47, 2020.

105. Yang Y, Qi S, Shi C, Han X, Yu J, Zhang L, Qin S and Gao Y: Identification of metastasis and prognosis-associated genes for serous ovarian cancer. Biosci Rep 40: BSR20194324, 2020.

106. Kuroki L and Guntupalli SR: Treatment of epithelial ovarian cancer. BMJ 371: m3773, 2020

107. Rooth C: Ovarian cancer: Risk factors, treatment and management. Br J Nurs 22: S23-S30, 2013.

108. Penson RT, Valencia RV, Cibula D, Colombo N, Leath CA III, Bidziński M, Kim JW, Nam JH, Madry R, Hernández C, et al: Olaparib versus nonplatinum chemotherapy in patients with platinum-sensitive relapsed ovarian cancer and a germline BRCA1/2 mutation (SOLO3): A randomized phase III trial J Clin Oncol 38: 1164-1174, 2020

109. Yadav G, Vashisht M, Yadav V and Shyam R: Molecular biomarkers for early detection and prevention of ovarian cancer-a gateway for good prognosis: A narrative review. Int J Prev Med 11: 135, 2020.

110. Chen HH, Zhou HJ, Wu GD and Lou XE: Inhibitory effects of artesunate on angiogenesis and on expressions of vascular endothelial growth factor and VEGF receptor KDR/flk-1. Pharmacology 71: 1-9, 2004.

111. Wang B, Hou D, Liu Q, Wu T, Guo H, Zhang X, Zou Y, Liu Z, Liu J, Wei J, et al: Artesunate sensitizes ovarian cancer cells to cisplatin by downregulating RAD51. Cancer Biol Ther 16 $1548-1556,2015$.

112. Fatehi Hassanabad A, Chehade R, Breadner D and Raphael J: Esophageal carcinoma: Towards targeted therapies. Cell Oncol (Dordr) 43: 195-209, 2020

113. Fan J, Liu Z, Mao X, Tong X, Zhang T, Suo C and Chen X: Global trends in the incidence and mortality of esophageal cancer from 1990 to 2017. Cancer Med 9: 6875-6887, 2020.
114. Shi R, Cui H, Bi Y, Huang X, Song B, Cheng C, Zhang L, Liu J, He C, Wang F, et al: Artesunate altered cellular mechanical properties leading to deregulation of cell proliferation and migration in esophageal squamous cell carcinoma. Oncol Lett 9: 2249-2255, 2015.

115. Eusebi LH, Telese A, Marasco G, Bazzoli F and Zagari RM: Gastric cancer prevention strategies: A global perspective. J Gastroenterol Hepatol 35: 1495-1502, 2020.

116. Niu PH, Zhao LL, Wu HL, Zhao DB and Chen YT: Artificial intelligence in gastric cancer: Application and future perspectives. World J Gastroenterol 26: 5408-5419, 2020

117. Zhou X, Sun WJ, Wang WM, Chen K, Zheng JH, Lu MD, Li PH and Zheng ZQ: Artesunate inhibits the growth of gastric cancer cells through the mechanism of promoting oncosis both in vitro and in vivo. Anticancer Drugs 24: 920-927, 2013.

118. Su T, Li F, Guan J, Liu L, Huang P, Wang Y, Qi X, Liu Z, Lu L and Wang D: Artemisinin and its derivatives prevent Helicobacter pylori-induced gastric carcinogenesis via inhibition of NF- $\mathrm{KB}$ signaling. Phytomedicine 63: 152968, 2019.

119. La Vecchia S and Sebastián C: Metabolic pathways regulating colorectal cancer initiation and progression. Semin Cell Dev Biol 98: 63-70, 2020.

120. Johdi NA and Sukor NF: Colorectal cancer immunotherapy: Options and strategies. Front Immunol 11: 1624, 2020.

121. Li LN, Zhang HD, Yuan SJ, Tian ZY, Wang L and Sun ZX Artesunate attenuates the growth of human colorectal carcinoma and inhibits hyperactive Wnt/beta-catenin pathway. Int J Cancer 121: 1360-1365, 2007.

122. Cui C, Feng H, Shi X, Wang Y, Feng Z, Liu J, Han Z, Fu J, $\mathrm{Fu} \mathrm{Z}$ and Tong $\mathrm{H}$ : Artesunate down-regulates immunosuppression from colorectal cancer Colon 26 and RKO cells in vitro by decreasing transforming growth factor $\beta 1$ and interleukin-10. Int Immunopharmacol 27: 110-121, 2015.

123. Bei Y, Chen X, Raturi VP, Liu K, Ye S, Xu Q and Lu M: Treatment patterns and outcomes change in early-stage non-small cell lung cancer in octogenarians and older: A SEER database analysis. Aging Clin Exp Res 33: 147-156, 2021

124. Huo KG, D'Arcangelo E and Tsao MS: Patient-derived cell line, xenograft and organoid models in lung cancer therapy. Transl Lung Cancer Res 9: 2214-2232, 2020

125. Xu K, Zhang C, Du T, Gabriel ANA, Wang X, Li X, Sun L, Wang N, Jiang X and Zhang Y: Progress of exosomes in the diagnosis and treatment of lung cancer. Biomed Pharmacother 134: 111111,2021

126. Rasheed SA, Efferth T, Asangani IA and Allgayer H: First evidence that the antimalarial drug artesunate inhibits invasion and in vivo metastasis in lung cancer by targeting essential extracellular proteases. Int J Cancer 127: 1475-1485, 2010.

127. Zhao Y, Jiang W, Li B, Yao Q, Dong J, Cen Y, Pan X, Li J, Zheng J, Pang X and Zhou H: Artesunate enhances radiosensitivity of human non-small cell lung cancer A549 cells via increasing NO production to induce cell cycle arrest at $\mathrm{G} 2 / \mathrm{M}$ phase. Int Immunopharmacol 11: 2039-2046, 2011.

128. Wang JS, Wang MJ, Lu X, Zhang J, Liu QX, Zhou D, Dai JG and Zheng $\mathrm{H}$ : Artesunate inhibits epithelial-mesenchymal transition in non-small-cell lung cancer (NSCLC) cells by down-regulating the expression of BTBD7. Bioengineered 11: 1197-1207, 2020

129. Chen X, Han K, Chen F, Wu C and Huang W: Effects of artesunate on the invasion of lung adenocarcinoma A549 cells and expression of ICAM-1 and MMP-9. Zhongguo Fei Ai Za Zhi 16: 567-571, 2013 (In Chinese)

130. Tong Y, Liu Y, Zheng H, Zheng L, Liu W, Wu J, Ou R, Zhang G, Li F, Hu M, et al: Artemisinin and its derivatives can significantly inhibit lung tumorigenesis and tumor metastasis through Wnt/3-catenin signaling. Oncotarget 7: 31413-31428, 2016.

131. Li W, Ma G, Deng Y, Wu Q, Wang Z and Zhou Q: Artesunate exhibits synergistic anti-cancer effects with cisplatin on lung cancer A549 cells by inhibiting MAPK pathway. Gene 766 : $145134,2021$.

132. Anwanwan D, Singh SK, Singh S, Saikam V and Singh R: Challenges in liver cancer and possible treatment approaches. Biochim Biophys Acta Rev Cancer 1873: 188314, 2020.

133. Cheng Z, Wei-Qi J and Jin D: New insights on sorafenib resistance in liver cancer with correlation of individualized therapy. Biochim Biophys Acta Rev Cancer 1874: 188382, 2020.

134. Hou J, Wang D, Zhang R and Wang H: Experimental therapy of hepatoma with artemisinin and its derivatives: In vitro and in vivo activity, chemosensitization, and mechanisms of action. Clin Cancer Res 14: 5519-5530, 2008. 
135. Jin M, Shen $X$, Zhao C, Qin X, Liu H, Huang L, Qiu Z and Liu Y: In vivo study of effects of artesunate nanoliposomes on human hepatocellular carcinoma xenografts in nude mice. Drug Deliv 20: 127-133, 2013.

136. Guragain D, Seubwai W, Kobayashi D, Silsinivanit A, Vaeteewoottacharn K, Sawanyawisuth K, Wongkham C, Wongkham S, Araki N and Cha'on U: Artesunate and chloroquine induce cytotoxic activity on cholangiocarcinoma cells via different cell death mechanisms. Cell Mol Biol (Noisy-le-grand) 64: 113-118, 2018.

137. Wang ZC, Liu Y, Wang H, Han QK and Lu C: Research on the relationship between artesunate and raji cell autophagy and apoptosis of burkitt's lymphoma and its mechanism. Eur Rev Med Pharmacol Sci 21: 2238-2243, 2017.

138. Chauhan AK, Min KJ and Kwon TK: RIP1-dependent reactive oxygen species production executes artesunate-induced cell death in renal carcinoma caki cells. Mol Cell Biochem 435: $15-24,2017$

139. Sarma B, Willmes C, Angerer L, Adam C, Becker JC, Kervarrec T, Schrama D and Houben R: Artesunate affects T antigen expression and survival of virus-positive merkel cell carcinoma. Cancers (Basel) 12: 919, 2020.

140. Zheng L and Pan J: The anti-malarial drug artesunate blocks Wnt/ $\beta$-catenin pathway and inhibits growth, migration and invasion of uveal melanoma cells. Curr Cancer Drug Targets 18: 988-998, 2018.
141. Wang Z, Wang C, Wu Z, Xue J, Shen B, Zuo W, Wang Z and Wang SL: Artesunate suppresses the growth of prostatic cancer cells through inhibiting androgen receptor. Biol Pharm Bull 40: 479-485, 2017.

142. Yang Y, Wu N, Wu Y, Chen H, Qiu J, Qian X, Zeng J, Chiu K, Gao Q and Zhuang J: Artesunate induces mitochondria-mediated apoptosis of human retinoblastoma cells by upregulating Kruppel-like factor 6. Cell Death Dis 10: 862, 2019.

143. Roh JL, Kim EH, Jang H and Shin D: Nrf2 inhibition reverses the resistance of cisplatin-resistant head and neck cancer cells to artesunate-induced ferroptosis. Redox Biol 11: 254-262, 2017.

144. Berköz M, Özkan-Yılmaz F, Özlüer-Hunt A, Krośniak M, Türkmen Ö, Korkmaz D and Keskin S: Artesunate inhibits melanoma progression in vitro via suppressing STAT3 signaling pathway. Pharmacol Rep 73: 650-663, 2021.

145. Mancuso RI, Foglio MA and Olalla Saad ST: Artemisinin-type drugs for the treatment of hematological malignancies. Cancer Chemother Pharmacol 87: 1-22, 2021.

This work is licensed under a Creative Commons Attribution-NonCommercial-NoDerivatives 4.0 International (CC BY-NC-ND 4.0) License. 